ARTICLE

\title{
Metal-free oxidative cross-coupling enabled practical synthesis of atropisomeric QUINOL and its derivatives
}

Peng-Ying Jiang ${ }^{1,3}$, Kai-Fang Fan ${ }^{1,3}$, Shaoyu Li (i] ${ }^{1,2 凶}$, Shao-Hua Xiang ${ }^{1,2}$ \& Bin Tan (iD) $^{1 凶}$

As an important platform molecule, atropisomeric QUINOL plays a crucial role in the development of chiral ligands and catalysts in asymmetric catalysis. However, efficient approaches towards QUINOL remain scarce, and the resulting high production costs greatly impede the related academic research as well as downstream industrial applications. Here we report a direct oxidative cross-coupling reaction between isoquinolines and 2-naphthols, providing a straightforward and scalable route to acquire the privileged QUINOL scaffolds in a metal-free manner. Moreover, a NHC-catalyzed kinetic resolution of QUINOL N-oxides with high selectivity factor is established to access two types of promising axially chiral Lewis base catalysts in optically pure forms. The utility of this methodology is further illustrated by facile transformations of the products into QUINAP, an iconic ligand in asymmetric catalysis.

\footnotetext{
${ }^{1}$ Department of Chemistry and Shenzhen Grubbs Institute, Guangdong Provincial Key Laboratory of Catalysis, Southern University of Science and Technology, Shenzhen, China. ${ }^{2}$ Academy for Advanced Interdisciplinary Studies, Southern University of Science and Technology, Shenzhen, China. ${ }^{3}$ These authors contributed equally: Peng-Ying Jiang, Kai-Fang Fan. ${ }^{凶}$ email: lisy@sustech.edu.cn; tanb@sustech.edu.cn
} 
T he capability to procure axially chiral biaryl skeletons with facility and efficiency has a major impact on advancing catalytic synthesis and material sciences owing to the widespread occurrence of these scaffolds in organocatalysts, ligands, and functional materials ${ }^{1-7}$. In particular, a streamlined access to a core framework from which modular installation of diversity is feasible for divergent construction of functional molecules, will greatly accelerate the development of these fields. 1-(Isoquinolin-1-yl)naphthalen-2-ol (QUINOL), a representative atropisomeric heterobiaryl, was described to function as reliable $\mathrm{N}-\mathrm{O}$ chelating ligand in the controlled polymerization of cyclic esters $^{8}$ while itself has been synthesized as a platform molecule to access other iconic ligands such as QUINAP ${ }^{9-13}$, QUINOX ${ }^{14,15}$ and IAN $^{16,17}$. A novel organic dye derived from QUINOL has also shown potential as vesicle stains in confocal fluorescence microscopy imaging (Fig. 1a) ${ }^{18}$. In view of these apparent utilities, concise and practical preparation of QUINOL and its derivatives becomes imperative, which remains an unaddressed synthetic challenge. The venerable Suzuki-Miyaura coupling reaction in the transition metal catalysis domain provides a reliable entry ${ }^{19,20}$ but the necessity to pre-functionalize the coupling partners has elongated step counts, hampering overall efficiency which culminates in the high production costs (Fig. 1b). This is reflected in the prices of some commercially available ligands derived from QUINOL. As displayed in Fig. 1a, enantiopure (S)-QUINAP costs about $2900 \mathrm{USD} / \mathrm{g}$ and even the racemic analog is sold at 1320 USD/g. The cost factor coupled with a lack of modularity has immensely restricted the related academic research as well as downstream industrial applications. These factors collectively illuminate the practical appeal to devise a unified strategy that constructs QUINOLs concisely, particularly in a cost-effective and metal-free manner.
One of the most convergent retrosynthetic disconnections of QUINOL frameworks would trace back to cross-dehydrogenative coupling of isoquinolines and 2-naphthols under oxidative conditions. It is remarkable that related method has never been accomplished, albeit the atom- and step-economy, probably due to the great disparity in redox potential between the two participating reactants. As an exceptional workaround for the limitations encountered in traditional oxidative-coupling, nucleophilic $\mathrm{C}-\mathrm{H}$ functionalization of electron-deficient $\mathrm{N}$-heteroarenes founded on $N$-activation mode has been realized, which encompasses a dearomatizing nucleophilic addition and a sequential oxidative rearomatization. Transition metals and organic reagents with strong Lewis acidity, inherent electrondeficient property as well as Lewis basicity of azaarenes are the key control elements that have been exploited for the success of this reaction type ${ }^{21-30}$. For instance, $\mathrm{ZnMe}_{2}$ was utilized by Cho and Baik in a highly regioselective alkylation of $N$-heteroarenes with 1,1-diborylalkanes ${ }^{23}$, while Kanai and Kuninobu developed a site-selective perfluoroalkylation of pyridines and quinolines with perfluoroalkyltrimethylsilane using a sterically bulky borane as the activator ${ }^{24}$. In addition, the $N$-acyl activation mode in Reissert reaction has been demonstrated to be one of the most efficient protocols for direct $\mathrm{C}-\mathrm{H}$ functionalization of six-membered heteroarenes by Fier $^{25,26}$ and McNally's group ${ }^{27-30}$. Building on these literature precedents, we envisaged the suitability of this activation mode to direct selective arylation of isoquinoline with 2-naphthol which will occur through a sequence of nucleophilic addition and oxidative re-aromatization to construct QUINOLs (Fig. 1c). The challenging aspect of this reaction design belongs to that of chemoselectivity; differentiation of multiple nucleophilic centers in 2-naphthol and several electrophilic sites in activated acyl isoquinolinium intermediate must be efficient to establish the

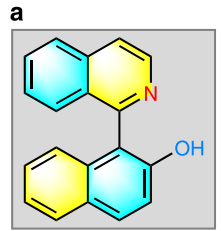

QUINOL

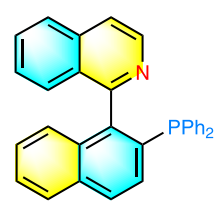

(S)-QUINAP: $2900 \$ / g$ (rac)-QUINAP: 1320 \$/g

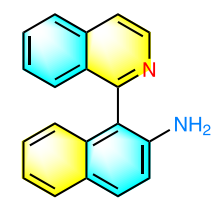

IAN: $553 \$ / 9$

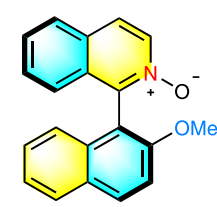

(S)-QUINOX

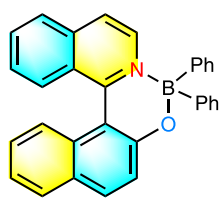

Fluorescent dyes

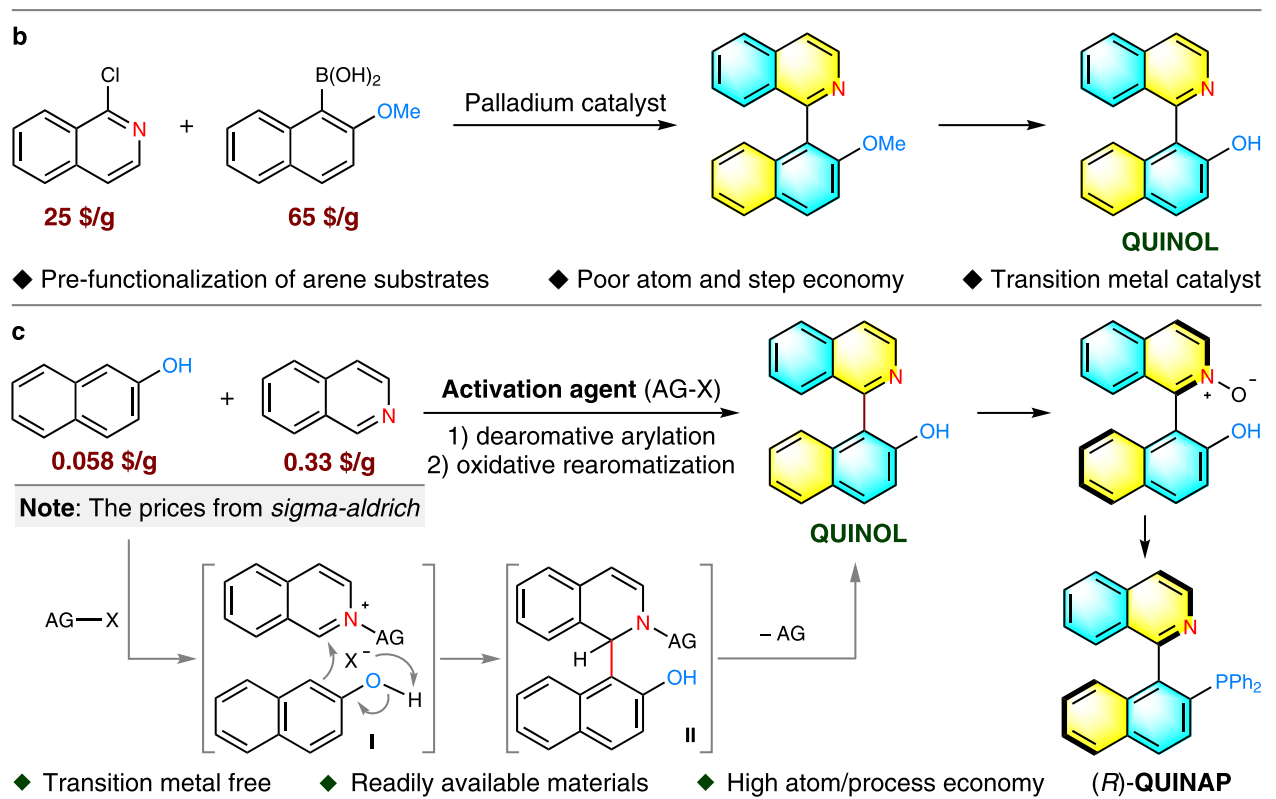

Fig. 1 The significance of QUINOL and its synthetic approaches. a QUINOL and representative biaryl isoquinoline structures. b Current strategy: transition-metal-catalyzed cross-coupling. c Our strategy: metal-free oxidative cross-coupling. 


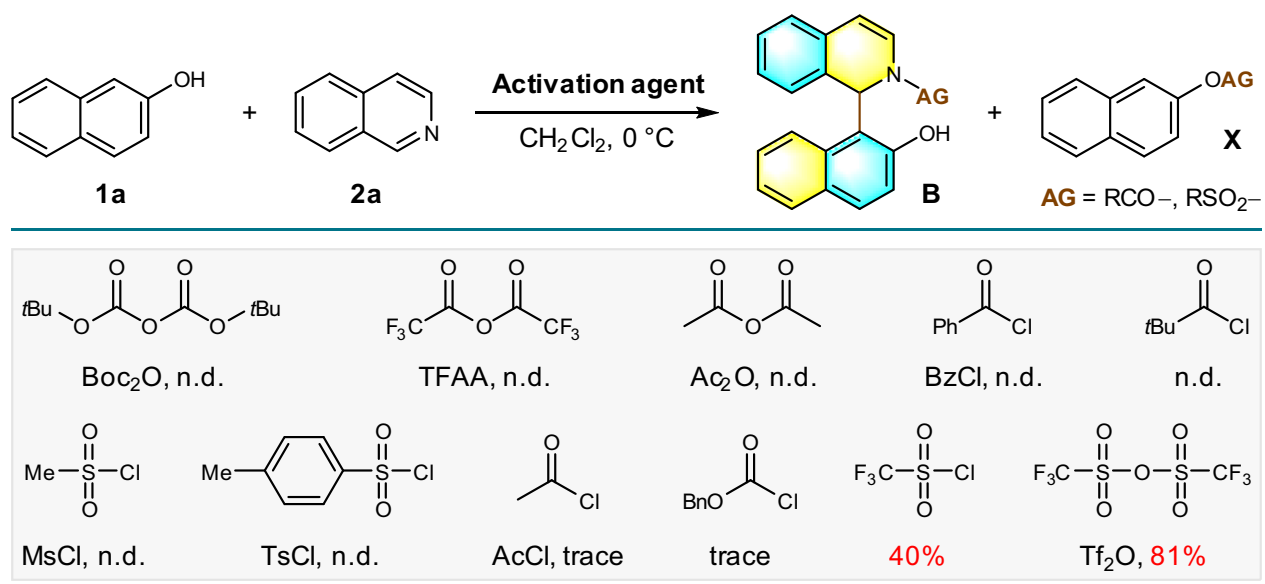

Fig. 2 Initial screening of activation agents. Conditions: acylation agent $(0.5 \mathrm{mmol})$ and $\mathbf{2 a}(1.25 \mathrm{mmol})$ were stirred in $\mathrm{CH}_{2} \mathrm{Cl}_{2}(4 \mathrm{~mL})$ for $0.5 \mathrm{~h}$ at $0{ }^{\circ} \mathrm{C}$. The solution of $\mathbf{1 a}(0.50 \mathrm{mmol})$ in $\mathrm{CH}_{2} \mathrm{Cl}_{2}(4 \mathrm{~mL})$ was then added and stirred for another $2 \mathrm{~h}$. Isolated yield was provided.

correct point of attachment. Consequently, the pursuit of an appropriate activation system becomes essential to realize this transformation.

Here, we show our successful endeavor to overcome the abovementioned challenges, opening up a practical synthetic pathway to structurally diversified QUINOLs by means of a metal-free oxidative cross-coupling reaction between isoquinoline and 2naphthol. On further development of a chiral NHC-catalyzed kinetic resolution of QUINOL N-oxides, two types of axially chiral QUINOL N-oxides, which are promising Lewis base catalysts can be readily accessible in optically pure forms. Importantly, scalable synthesis and useful transformation have demonstrated the utility of this methodology.

\section{Results}

Reaction condition optimization. Initiating our investigations, the influence of the activation reagents on dearomative arylation step was examined in a model coupling reaction of 2-naphthol 1a and isoquinoline $\mathbf{2 a}$ (Fig. 2). In the excess of isoquinoline, the first attempt with $\mathrm{Boc}_{2} \mathrm{O}$ in $\mathrm{CH}_{2} \mathrm{Cl}_{2}$ at $0{ }^{\circ} \mathrm{C}$ only afforded the byproduct from the esterification of 2-naphthol. Evaluation of a panel of acyl chloride and anhydride including TFAA, $\mathrm{Ac}_{2} \mathrm{O}, \mathrm{BzCl}$, $\mathrm{MsCl}, \mathrm{TsCl}$, and pivaloyl chloride has resulted in similar reaction outcomes. The first hint of success came when a trace amount of target compound $\mathbf{B}$ was detected by LCMS in reactions that employed $\mathrm{AcCl}$ or benzyl chloroformate. Following investigations revealed that trifluoromethanesulfonyl chloride could impart major improvement in reaction efficiency to provide B in $40 \%$ isolated yield. Trifluoromethanesulfonic anhydride $\left(\mathrm{Tf}_{2} \mathrm{O}\right)$ proved to be the optimal activator for selective formation of the $\mathrm{C}-\mathrm{C}$ bond under this set of conditions and delivered the desired product in $81 \%$ yield, along with $10 \%$ yield of esterification byproduct.

After a simple workup, intermediate $\mathbf{B}$ could be readily converted to the target QUINOL 3a in nearly quantitative yield by treatment with $\mathrm{K}_{2} \mathrm{CO}_{3}$ in DMSO at $60^{\circ} \mathrm{C}$ for $3 \mathrm{~h}$ (Fig. 3, entry $1)$. The identity of $\mathbf{3 a}$ was confirmed by single-crystal $\mathrm{X}$-ray diffraction analysis (CCDC 2005905). This achievement encouraged us to investigate other reaction parameters of the first arylation step to further enhance the overall effectiveness of this cross-coupling reaction. An increased loading of 2-naphthol (Fig. 3, entry 2) failed to suppress the side reactions, whereas switching the reaction medium to chloroform, toluene, ethyl acetate or diethyl ether did not improve the outcomes as well (Fig. 3, entries 3-6). Given $\mathrm{H}_{2} \mathrm{O}$ adduct was identified in LCMS during the initial optimization process, we reckoned that the moisture in the reaction system could be detrimental to the productive reaction pathway. As such, a series of additives known to sequester moisture were included. The addition of $\mathrm{Mg}_{2} \mathrm{SO}_{4}$ did not bring about any positive effect in terms of product yield (Fig. 3, entry 7) while $5 \AA$ molecular sieve successfully obstructed the $O$-acylation thus raised the product yield to $89 \%$ (Fig. 3, entry 8). Interestingly, competitive esterification of 2-naphthol was inhibited altogether when at lower reaction temperatures (Fig. 3, entries 9-11) with quantitative yield being secured at -50 or $-78^{\circ} \mathrm{C}$. The introduction of an exogenous base such as $\mathrm{Et}_{3} \mathrm{~N}$ or $\mathrm{NaHCO}_{3}$ has eroded the chemoselectivity instead (Fig. 3, entries 12 and 13).

Substrate scope. Having identified the optimal reaction parameters for the model coupling, we turned to probe the generality of this set of conditions. As shown in Fig. 4, a wide range of substrates with different substituents attached in varied patterns were well tolerated to form target products in high to excellent yields without chromatographic purification of the intermediates. Firstly, electronic variations on 2-naphthol substrates posed limited influence on the reactivity. Substrates with electrondonating and electron-withdrawing groups furnished the corresponding QUINOLs (3b-3p) in more than $80 \%$ yields within similar reaction duration, except $\mathbf{3 d}$ and $\mathbf{3 p}$. The slight decrease in the yields of $\mathbf{3 k}, \mathbf{3 l}$ and $\mathbf{3 m}$ may be ascribed to the poor solubility of reacting substrates. Notably, the tolerance of ester, cyano, aldehyde, and acetyl groups signified the potential chemical manipulations to introduce other useful functionalities onto product scaffold (Fig. 4b). Next, the conditions were studied with respect to variations on isoquinoline where all selected substrates generally furnished the expected products $\mathbf{3 q}-\mathbf{3} \mathbf{x}$ in high efficiency (Fig. 4b). It could be discerned that the coupling efficacy of electron-poor isoquinolines ( $\mathbf{3 v}$ and $\mathbf{3 w}$ ) was lower compared to the more electron-rich analogs $(\mathbf{3 q}$ and $\mathbf{3 r}$ ) or those carrying halide moiety $(\mathbf{3 s}-\mathbf{3 u})$. Moreover, the 3 -position substituent of isoquinoline posed a negative effect on the transformation $(3 \mathbf{x})$. To enrich the structural diversity of QUINOL framework, a wide spectrum of di-substituted QUINOL derivatives were synthesized smoothly using the same set of conditions (3y-3aj). It is worth pointing out that the ability to introduce substituents at both $7,7^{\prime}$ positions of QUINOL backbone provides the flexibility to adjust the dihedral angle of the biaryl motifs, which is a strong asset in the design and fine-tuning of ligands or organocatalysts. More importantly, bromo substituent which was commonly 


\begin{tabular}{|c|c|c|c|c|}
\hline Entry & Solvent & Additive & Temp $\left({ }^{\circ} \mathrm{C}\right)$ & Yield $(\%)^{b}$ \\
\hline 1 & $\mathrm{CH}_{2} \mathrm{Cl}_{2}$ & - & 0 & 80 \\
\hline $2^{c}$ & $\mathrm{CH}_{2} \mathrm{Cl}_{2}$ & - & 0 & 82 \\
\hline 3 & $\mathrm{CHCl}_{3}$ & - & 0 & 45 \\
\hline 4 & toluene & - & 0 & 34 \\
\hline 5 & EtOAc & - & 0 & 35 \\
\hline 6 & $\mathrm{Et}_{2} \mathrm{O}$ & - & 0 & 12 \\
\hline 7 & $\mathrm{CH}_{2} \mathrm{Cl}_{2}$ & $\mathrm{MgSO}_{4}$ & 0 & 80 \\
\hline 8 & $\mathrm{CH}_{2} \mathrm{Cl}_{2}$ & $5 \AA ̊ M S$ & 0 & 89 \\
\hline 9 & $\mathrm{CH}_{2} \mathrm{Cl}_{2}$ & $5 \AA \mathrm{MS}$ & -30 & 91 \\
\hline 10 & $\mathrm{CH}_{2} \mathrm{Cl}_{2}$ & $5 \AA \mathrm{MS}$ & -50 & 98 \\
\hline 11 & $\mathrm{CH}_{2} \mathrm{Cl}_{2}$ & $5 \AA \mathrm{MS}$ & -78 & 98 \\
\hline $12^{\mathrm{d}}$ & $\mathrm{CH}_{2} \mathrm{Cl}_{2}$ & $5 \AA ̊ M S$ & -78 & 49 \\
\hline $13^{\mathrm{e}}$ & $\mathrm{CH}_{2} \mathrm{Cl}_{2}$ & $5 \AA \mathrm{MS}$ & -78 & 44 \\
\hline
\end{tabular}

Fig. 3 Optimization of the conditions ${ }^{\mathbf{a}}$. aReaction conditions: To a Schlenk tube was added additive ( 0 or $\left.250 \mathrm{mg}\right), \mathbf{2 a}(1.25 \mathrm{mmol}), \mathrm{CH}_{2} \mathrm{Cl} 2(4 \mathrm{~mL})$ and $\mathrm{Tf}_{2} \mathrm{O}(0.5 \mathrm{mmol})$ successively. After stirring at specified temperature for $0.5 \mathrm{~h}$, a solution of $\mathbf{1 a}(0.5 \mathrm{mmol})$ in $\mathrm{CH}_{2} \mathrm{Cl}_{2}(4 \mathrm{~mL})$ was added. The reaction

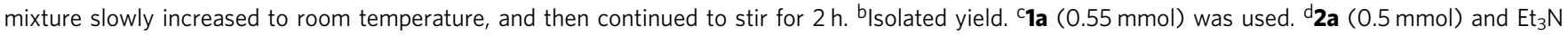
(1.5 eq.) were used. e2a $(0.5 \mathrm{mmol})$ and $\mathrm{NaHCO}_{3}(1.5$ eq.) were used.

incompatible with conventional palladium-catalyzed SuzukiMiyaura cross-couplings was left unscathed on both coupling partners under our metal-free reaction conditions $(\mathbf{3 i}, \mathbf{3 s}-\mathbf{3 u}$, and 3y-3ah). The orthogonality of the present method to classic cross-coupling chemistry would permit downstream functionalization and further diversification of QUINOL scaffolds via these robust coupling protocols.

The untapped utilitarian potential of these structurally diverse QUINOL analogs in asymmetric catalysis provides a strong incentive to resolve the racemates using kinetic resolution. Initial efforts to isolate the individual enantiomers by preparative chiral HPLC revealed the low configurational stability of the stereogenic axis in these scaffolds. We sought to continue the endeavor through synthesizing the more stable QUINOL N-oxide derivatives, which could be easily achieved from the oxidation of prepared QUINOLs with DMDO. Several relevant works have served as important precedents for our approach. The preparation of axially chiral QUINOL scaffolds via kinetic resolution is pioneered by You and $\mathrm{Gu}$ in 2014, where Pd(II)-catalyzed direct $\mathrm{C}-\mathrm{H}$ iodination forged axially chiral isoquinoline $\mathrm{N}$-oxides with moderate enantioselectivity ${ }^{31}$. A biocatalytic dynamic kinetic resolution enabled by configurational lability of precursors was then prominently disclosed by Clayden and Turner ${ }^{32}$. Diversely, Matsubara and Asano attained a highly enantioselective aromatic electrophilic bromination of 1-(3-hydroxyphenyl)isoquinoline 2oxides to construct axially chiral isoquinoline $\mathrm{N}$-oxides by using a bifunctional catalyst ${ }^{33}$. Recognizing the structural characteristic of our QUINOL N-oxide products, the contributions from Zhao $^{34}$ and $\mathrm{Wang}^{35}$ on enantioselective kinetic resolution of NOBIN analogs via chiral $N$-heterocyclic carbene (NHC) catalyzed acylation reactions inspired our formulation of an analogous strategy to obtain the axially chiral QUINOL derivatives in enantioenriched forms.

Kinetic resolution of QUINOL N-oxides. An interrogation of different NHC precatalysts for this kinetic resolution process has demonstrated the sensitivity of enantioselectivity outcome towards the identity of aryl group on the triazolium moiety where sterically hindered ones were found unfavorable. C7 which possesses a nitro group on the other aryl group, was subsequently identified as the lead precatalyst. The poor solubility of QUINOL $\mathrm{N}$-oxide in most common solvents put an additional constraint on stereochemical control. Eventually, this was effectively addressed through employing a binary mixture of $\mathrm{CH}_{2} \mathrm{Cl}_{2}$ and DMSO (for more detailed reaction optimization of the kinetic resolution, see Supplementary Table 3). We also investigated the generality of this NHC-catalyzed kinetic resolution strategy (Fig. 5) and found it to be pleasingly broad. Most racemic QUINOL N-oxides could be stereochemically enriched to 85$>99 \%$ ee and isolated in $40-44 \%$ yield. Particularly noteworthy is that the acylation products 5 and the remaining $(R)-4$ could be obtained with high yields and good ee values simply by adjusting the loading of aldehydes. Under the standard conditions $(0.65$ equiv of aldehyde), $(R)-\mathbf{4 a}$ was produced in $43 \%$ yield with $>99 \%$ ee while the acylation product $\mathbf{5}$ a was afforded in $45 \%$ yield and 93\% ee using 0.5 equiv. of aldehyde (Fig. 5). The absolute configuration of $(R)-4$ a was determined by X-ray crystallographic analysis (CCDC 2046559), and those of other QUINOL N-oxides displayed in Fig. 5 were assigned by analogy.

Preparative synthesis and transformations. The practicality of the methodology in terms of scalability was first testified with a decagram-scale synthesis of QUINOL product performed under the optimal reaction conditions (Fig. 6a). QUINOL 3a was effortlessly generated in $92 \%$ yield and 12.5 gram when the standard reaction was performed on a $50 \mathrm{mmol}$ scale. The procedural simplicity (i.e., post-reaction treatment and rapid recrystallization of product) holds promises for industrial-scale production. The streamlined synthesis of QUINAP from QUINOL 3a was also scalable and an overall yield of $79 \%$ was obtained over two steps. Subsequently, optically active $(R)$-QUINAP was efficiently synthesized in four steps using enantiopure 


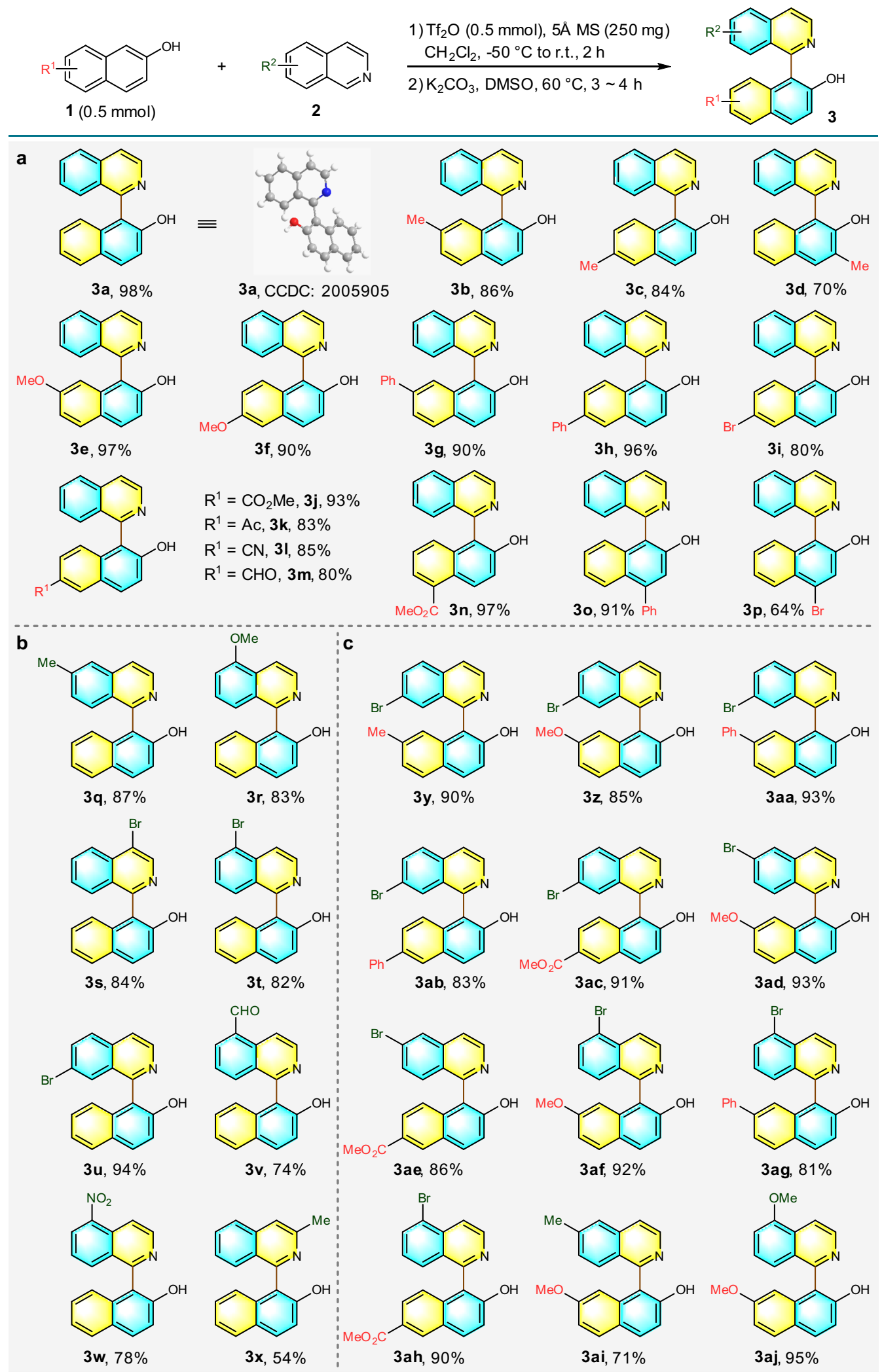

Fig. 4 Substrate scope of the oxidative cross-coupling of 2-naphthols and isoquinolines. a Scope of 2-naphthalenol. b Scope of isoquinoline. c Modification of both coupling partners. 

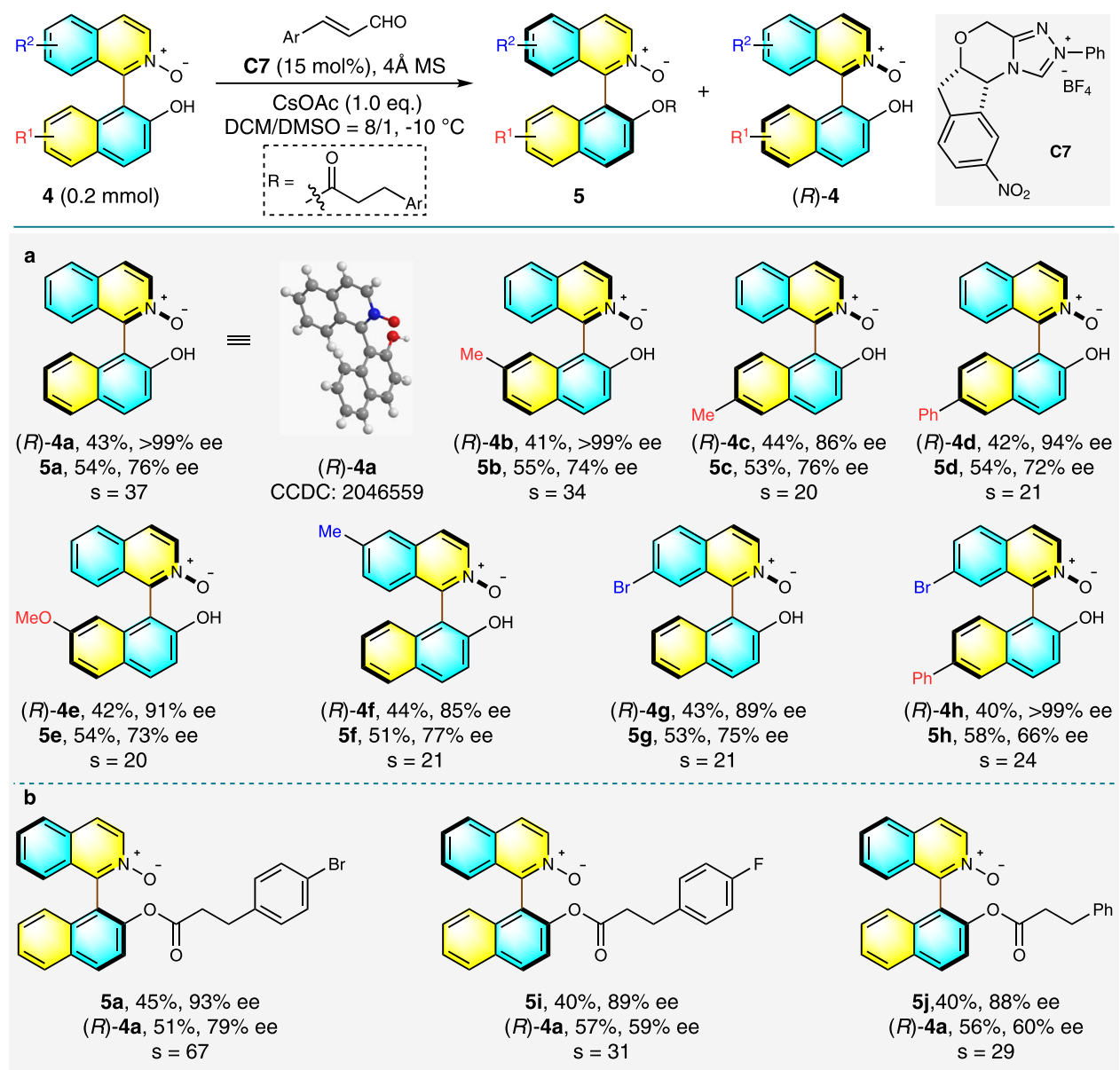

Fig. 5 Substrate scope of NHC-catalyzed kinetic resolution of QUINOL N-oxides. a $\mathrm{Ar}=4-\mathrm{Br}-\mathrm{Ph}(0.65$ eq. aldehyde). $\mathbf{b}$ with 0.5 eq. aldehydes.

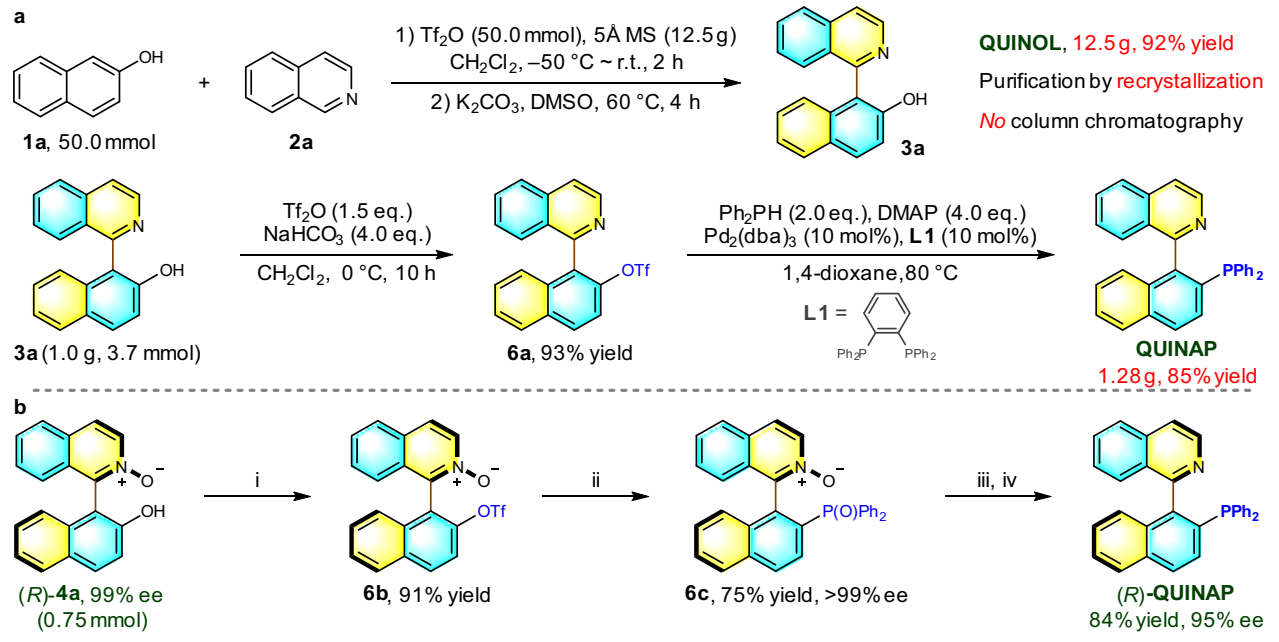

Fig. 6 Preparative synthesis and transformations. a Gram-scale synthesis of (rac)-QUINOL and (rac)-QUINAP. b Synthesis of ( $R$ )-QUINAP from ( $R$ )QUINOL N-oxide. Reaction conditions: i $\mathrm{Tf}_{2} \mathrm{O}$ (1.05 eq.), DMAP (2.0 eq.), $\mathrm{CH}_{2} \mathrm{Cl}_{2}$, r.t., 10 h; ii diphenylphosphine oxide (2.0 eq.), Pd(OAc) 2 (12 mol\%), L1 (24 mol\%), DIPEA (5.5 eq.), DMSO, $110^{\circ} \mathrm{C}, 24 \mathrm{~h}$; iii bis(pinacolato)diboron (1.0 eq.), diglyme, $70{ }^{\circ} \mathrm{C}, 12 \mathrm{~h}$; iv $\mathrm{HSiCl}_{3}\left(10.0\right.$ eq.), DIPEA ( 40.0 eq.), toluene, $70{ }^{\circ} \mathrm{C}, 4 \mathrm{~h}$.

$(R)$-QUINOL N-oxide as the starting material with routine transformations (Fig. 6b).

Controlled experiments and proposed reaction processes. Guided by our continuous understanding of the construction of axially chiral compounds with 2 -naphthol as the nucleophile ${ }^{36}$, a plausible mechanistic pathway was rationalized as outlined in Fig. 7. This coupling reaction is initiated when $\mathrm{Tf}_{2} \mathrm{O}$ activates the isoquinoline $\mathbf{2 a}$ in the form acyl isoquinolinium $\mathbf{A}$. The nucleophilic 2-naphthol 1a then adds to the iminium carbon of activated species A selectively from $\mathrm{C} 1$ position, as simultaneously promoted by the proton abstraction from hydroxyl group by the 
a
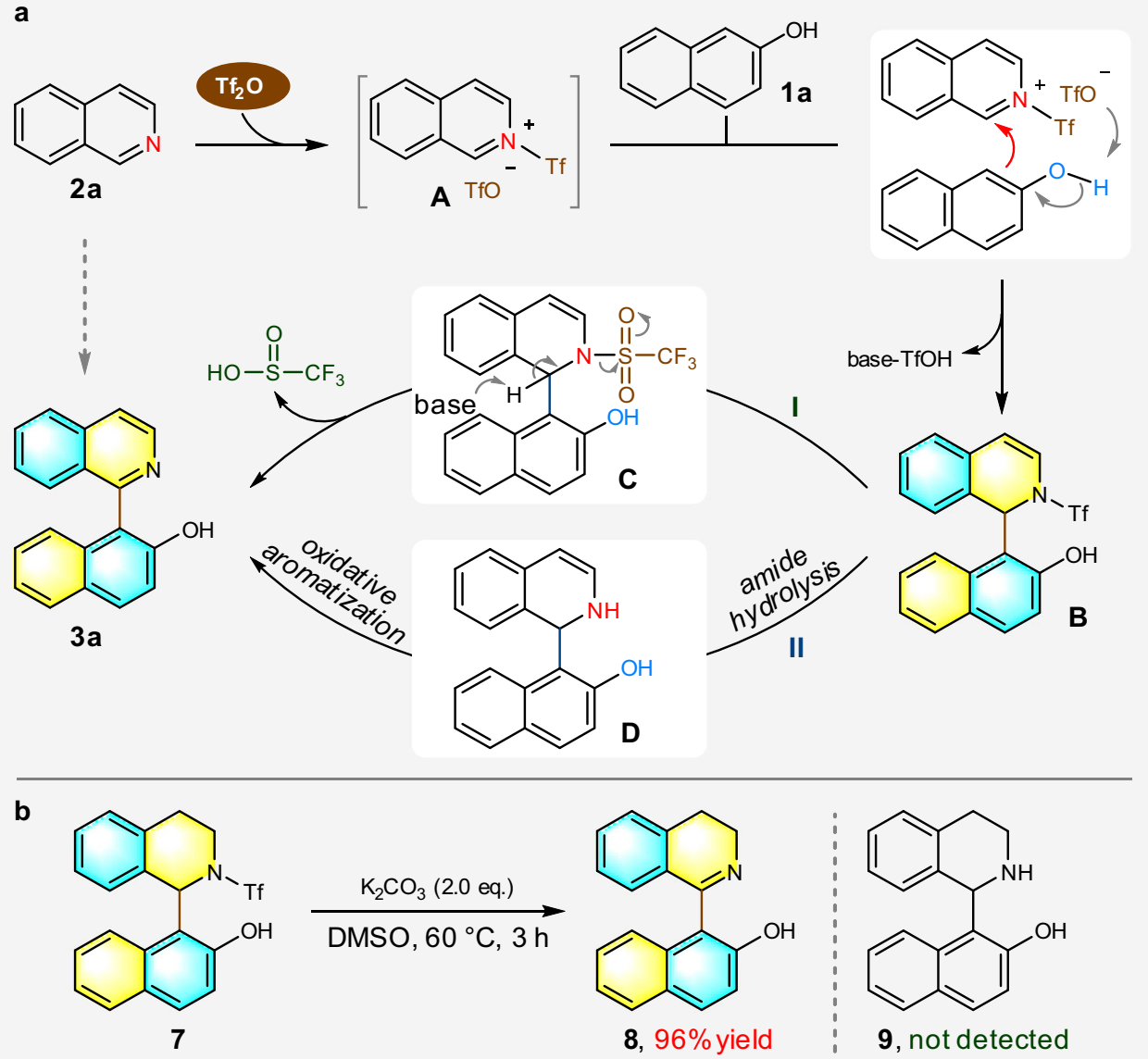

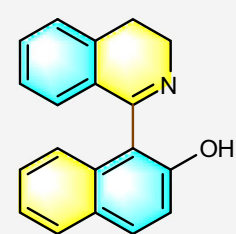

8, $96 \%$ yield<smiles></smiles>

Fig. 7 Proposed reaction mechanism and control experiment. a Proposed reaction pathway. b Control experiment.

proximal triflate anion; tautomerization at naphthol ring then gives rise to dearomatized intermediate B. Such intermolecular synergistic activation-addition events may play a crucial role to overcome the otherwise challenging chemoselectivity issue resulting from competitive nucleophilic and electrophilic sites present on these reacting partners. Finally, an oxidative aromatization process delivers the target product QUINOL 3a along with the removal of $\mathrm{Tf}$ group. Noteworthily, there are two possible pathways for the last step, namely, base-promoted redox process (Fig. 7a, pathway I) or amide hydrolysis-aromatizationdriven oxidation cascade process (Fig. 7a, pathway II). To obtain more insights into this step, a control experiment was designed. In light of the powerful driving force for aromatization in intermediate $\mathbf{D}$, it should be infeasible to capture such highly active species. Therefore, the hydrogenation product $\mathbf{7}$ of intermediate $\mathbf{B}$ was prepared as the starting material for mechanistic investigation. As displayed in Fig. 7b, the treatment of 7 under the standard conditions provided product $\mathbf{8}$ in $96 \%$ yield with no formation of product 9 . This lent indirect evidence to rule out the possibility of pathway II and indicated that $\mathrm{Tf}_{2} \mathrm{O}$ is not only an activator of isoquinoline but also an oxidant for the oxidative aromatization process.

\section{Discussion}

In conclusion, a metal-free oxidative cross-coupling reaction between isoquinoline and 2-naphthol has been discovered to deliver structurally diversified QUINOLs. This protocol features desirable blend of high yield, exclusive chemoselectivity, robust functional group tolerance, and step economy for the construction of privileged QUINOL derivatives. Moreover, the low cost of raw materials, ease of purification, and decagram-scale preparation portend the adaptability to process settings. For illustration, a streamlined and large-scale synthesis of QUINAP from QUINOL product was demonstrated which presents superiority in terms of production cost. Following a chiral NHCcatalyzed kinetic resolution, two types of axially chiral QUINOL $\mathrm{N}$-oxides which are promising Lewis base catalysts were readily accessible in enantioenriched forms. Derivatization into $(R)$ QUINAP was also accomplished with standard chemical transformations, thus providing a practical method to access these important frameworks.

\section{Methods}

Procedure for the enantioselective synthesis of $\mathbf{3}$. To a dried Schlenk tube was added $5 \AA$ molecular sieve $(250 \mathrm{mg})$, isoquinoline $2(1.25 \mathrm{mmol})$, and $\mathrm{CH}_{2} \mathrm{Cl}_{2}$ $(4 \mathrm{~mL})$ under argon atmosphere. After the solution was cooled to $-50{ }^{\circ} \mathrm{C}, \mathrm{Tf}_{2} \mathrm{O}$ $(0.5 \mathrm{mmol})$ was added through a microsyringe and stirred for half an hour until white solid salts appeared. After adding the solution of 2-naphthol $\mathbf{1}(0.5 \mathrm{mmol})$ in $\mathrm{CH}_{2} \mathrm{Cl}_{2}(4 \mathrm{~mL})$, the reaction was slowly raised to room temperature and then continued to stir for $2 \mathrm{~h}$. The molecular sieve was removed by filtration, and the resulting filtrate was washed successively with $3 \mathrm{M} \mathrm{HCl}$, saturated sodium bicarbonate, and brine, and then dried over anhydrous $\mathrm{Na}_{2} \mathrm{SO}_{4}$. After evaporating the solvent under reduced pressure, the resulting intermediate $\mathbf{B}$ was dissolved in $4 \mathrm{~mL}$ of DMSO, and $\mathrm{K}_{2} \mathrm{CO}_{3}(138 \mathrm{mg}, 1.0 \mathrm{mmol})$ was then added. The mixture was stirred at $60^{\circ} \mathrm{C}$ for about $3-4 \mathrm{~h}$ until the intermediate was consumed completely. The reaction system was adjusted to weakly acidic with $3 \mathrm{M} \mathrm{HCl}$, and then to weakly basic with saturated sodium bicarbonate. The mixture was diluted with $20 \mathrm{~mL}$ of water, and extracted with EA $(20 \mathrm{~mL} \times 3)$. The combined organic phase was washed with brine $(20 \mathrm{~mL} \times 2)$, dried over anhydrous $\mathrm{Na}_{2} \mathrm{SO}_{4}$, and concentrated to afford the crude product, which was purified by column chromatography eluted with PE/EA (10/1 to $3 / 1)$ to afford the pure product. 
Procedure for the kinetic resolution of 4. Under argon atmosphere, substrate 4 (0.2 mmol), cinnamaldehyde $(0.13 \mathrm{mmol}, 0.65 \mathrm{eq}$.), C7 (13 mg, $0.03 \mathrm{mmol})$, activated $4 \AA \mathrm{MS}(400 \mathrm{mg})$, solvent $\left(18 \mathrm{~mL}, \mathrm{CH}_{2} \mathrm{Cl}_{2} / \mathrm{DMSO}=8 / 1\right)$, and $\mathrm{CsOAc}$ $(0.2 \mathrm{mmol}, 1.0$ eq.) were added sequentially to a dried Schlenk flask. The mixture was stirred at $-10{ }^{\circ} \mathrm{C}$ until the aldehyde was completely consumed (monitored by TLC). After removing the molecular sieve by filtration, the obtained filtrate was diluted with $30 \mathrm{~mL}$ of water, and then extracted with DCM $(30 \mathrm{~mL} \times 3)$. The combined organic phase was washed with $30 \mathrm{~mL}$ brine, dried over anhydrous $\mathrm{Na}_{2} \mathrm{SO}_{4}$, and concentrated to afford crude products, which were purified by column chromatography eluted with DCM/EA (20/1 to 1/1) to afford the pure products 5 and $(R)-4$.

\section{Data availability}

The X-ray crystallographic coordinates for structures reported in this Article have been deposited at the Cambridge Crystallographic Data Centre (CCDC), under deposition numbers CCDC 2005905, CCDC 2046559. These data can be obtained free of charge from The Cambridge Crystallographic Data Centre via http://www.ccdc.cam.ac.uk/ data_request/cif.

Received: 7 December 2020; Accepted: 5 March 2021; Published online: 22 April 2021

\section{References}

1. Cepanec, I. Synthesis of Biaryls (2004).

2. Ackermann, L. Modern Arylation Methods (2009).

3. Zhou, Q.-L. Privileged Chiral Ligands and Catalysts (2011).

4. Hassan, J., Sévignon, M., Gozzi, C., Schulz, E. \& Lemaire, M. Aryl-aryl bond formation one century after the discovery of the Ullmann reaction. Chem. Rev. 102, 1359-1469 (2002).

5. Bringmann, G. et al. Atroposelective synthesis of axially chiral biaryl compounds. Angew. Chem. Int. Ed. 44, 5384-5427 (2005).

6. Surry, D. S. \& Buchwald, S. L. Biaryl phosphane ligands in palladiumcatalyzed amination. Angew. Chem. Int. Ed. 47, 6338-6361 (2008).

7. Wu, J.-S., Cheng, S.-W., Cheng, Y.-J. \& Hsu, C.-S. Donor-acceptor conjugated polymers based on multifused ladder-type arenes for organic solar cells. Chem. Soc. Rev. 44, 1113-1154 (2015).

8. Howard, R. H. et al. Synthesis and structures of complexes with axially chiral isoquinolinyl-naphtholate ligands. Dalton Trans. 2009, 8667-8682 (2009).

9. Chen, C., Li, X. \& Schreiber, S. L. Catalytic asymmetric [3+2] cycloaddition of azomethine ylides. Development of a versatile stepwise, three-component reaction for diversity-oriented synthesis. J. Am. Chem. Soc. 125, 10174-10175 (2003).

10. Gommermann, N., Koradin, C., Polborn, K. \& Knochel, P. Enantioselective, copper(I)-catalyzed three-component reaction for the preparation of propargylamines. Angew. Chem. Int. Ed. 42, 5763-5766 (2003).

11. Miura, T., Yamauchi, M., Kosaka, A. \& Murakami, M. Nickel-catalyzed regio- and enantioselective annulation reactions of 1,2,3,4-benzothiatriazine1,1(2H)-dioxides with allenes. Angew. Chem. Int. Ed. 49, 4955-4957 (2010).

12. Lim, A. D., Codelli, J. A. \& Reisman, S. E. Catalytic asymmetric synthesis of highly substituted pyrrolizidines. Chem. Sci. 4, 650-654 (2013).

13. Rokade, B. V. \& Guiry, P. J. Axially chiral $P, N$-ligands: some recent twists and turns. ACS Catal. 8, 624-643 (2018).

14. Malkov, A. V., Dufková, L., Farrugia, L. \& Kočovský, P. Quinox, a quinolinetype $\mathrm{N}$-oxide, as organocatalyst in the asymmetric allylation of aromatic aldehydes with allyltrichlorosilanes: the role of arene-arene interactions. Angew. Chem. Int. Ed. 42, 3674-3677 (2003).

15. Malkov, A. V. et al. On the mechanism of asymmetric allylation of aldehydes with allyltrichlorosilanes catalyzed by QUINOX, a chiral isoquinoline $N$ oxide. J. Am. Chem. Soc. 130, 5341-5348 (2008).

16. Cortright, S. B. \& Johnston, J. N. IAN-amines: direct entry to a chiral $\mathrm{C}_{2^{-}}$ symmetric zirconium(IV) $\beta$-diketimine complex. Angew. Chem. Int. Ed. 41, 345-348 (2002).

17. Cortright, S. B., Huffman, J. C., Yoder, R. A., Coalter, J. N. \& Johnston, J. N. IAN amines: chiral $C_{2}$-symmetric zirconium(IV) complexes from readily modified axially chiral $C_{1}$-symmetric $\beta$-diketimines. Organometallics 23, 2238-2250 (2004).

18. Pais, V. F. et al. Red-emitting tetracoordinate organoboron chelates: synthesis, photophysical properties, and fluorescence microscopy. J. Org. Chem. 81, 9605-9611 (2016).

19. Alcock, N. W., Brown, J. M. \& Hulmes, D. I. Synthesis and resolution of 1-(2diphenylphosphino-l-naphthyl)isoquinoline; a P-N chelating ligand for asymmetric catalysis. Tetrahedron 4, 743-756 (1993).
20. Lim, C. W. et al. Practical preparation and resolution of 1-(2'diphenylphosphino-1'-naphthyl)isoquinoline: a useful ligand for catalytic asymmetric synthesis. Org. Proc. Res. Dev. 7, 379-384 (2003).

21. Murakami, K., Yamada, S., Kaneda, T. \& Itami, K. C-H functionalization of azines. Chem. Rev. 117, 9302-9332 (2017)

22. Fier, P. S. \& Hartwig, J. F. Selective C-H fluorination of pyridines and diazines inspired by a classic amination reaction. Science 342, 956-960 (2013).

23. Jo, W. et al. $\mathrm{ZnMe}_{2}$-mediated, direct alkylation of electron-deficient N-heteroarenes with 1,1-diborylalkanes: scope and mechanism. J. Am. Chem. Soc. 142, 13235-13245 (2020).

24. Nagase, M., Kuninobu, Y. \& Kanai, M. 4-position-selective C-H perfluoroalkylation and perfluoroarylation of six-membered heteroaromatic compounds. J. Am. Chem. Soc. 138, 6103-6106 (2016).

25. Fier, P. S. A bifunctional reagent designed for the mild, nucleophilic functionalization of pyridines. J. Am. Chem. Soc. 139, 9499-9502 (2017).

26. Fier, P. S., Kim, S. \& Cohen, R. D. A multifunctional reagent designed for the site-selective amination of pyridines. J. Am. Chem. Soc. 142, 8614-8618 (2020).

27. Hilton, M. C., Dolewski, R. D. \& McNally, A. Selective functionalization of pyridines via heterocyclic phosphonium salts. J. Am. Chem. Soc. 138, 13806-13809 (2016).

28. Dolewski, R. D., Fricke, P. J. \& McNally, A. Site-selective switching strategies to functionalize polyazines. J. Am. Chem. Soc. 140, 8020-8026 (2018).

29. Levy, J. N., Alegre-Requena, J. V., Liu, R., Paton, R. S. \& McNally, A. Selective halogenation of pyridines using designed phosphine reagents. J. Am. Chem. Soc. 142, 11295-11305 (2020).

30. Hilton, $\mathrm{M}$. C. et al. Heterobiaryl synthesis by contractive $\mathrm{C}-\mathrm{C}$ coupling via $\mathrm{P}$ (V) intermediates. Science 362, 799-804 (2018).

31. Gao, D.-W., Gu, Q. \& You, S.-L. Pd(II)-catalyzed intermolecular direct C-H bond iodination: an efficient approach toward the synthesis of axially chiral compounds via kinetic resolution. ACS Catal. 4, 2741-2745 (2014).

32. Staniland, S. et al. Biocatalytic dynamic kinetic resolution for the synthesis of atropisomeric biaryl N-oxide lewis base. Catal. Angew. Chem. Int. Ed. 55, 10755-10759 (2016)

33. Miyaji, R., Asano, K. \& Matsubara, S. Bifunctional organocatalysts for the enantioselective synthesis of axially chiral isoquinoline $\mathrm{N}$-oxides. J. Am. Chem. Soc. 137, 6766-6769 (2015).

34. Lu, S., Poh, S. B. \& Zhao, Y. Kinetic resolution of 1,1'-biaryl-2,2'-diols and amino alcohols through NHC-catalyzed atroposelective acylation. Angew. Chem. Int. Ed. 53, 11041-11045 (2014).

35. Yang, G., Guo, D., Meng, D. \& Wang, J. NHC-catalyzed atropoenantioselective synthesis of axially chiral biaryl amino alcohols via a cooperative strategy. Nat. Commun. 10, 3062-3067 (2019).

36. Wang, Y.-B. \& Tan, B. Construction of axially chiral compounds via asymmetric organocatalysis. Acc. Chem. Res. 51, 534-547 (2018).

\section{Acknowledgements}

We are grateful for financial support from the National Natural Science Foundation of China (Nos. 21702092, 21772081, 21825105, and 21901105), Guangdong Provincial Key Laboratory of Catalysis (No. 2020B121201002), Guangdong Innovative Program (2019BT02Y335), Shenzhen Special Funds (JCYJ20190812112603598, JCYJ20180305123508258, and JCYJ20170412151701379), Shenzhen Nobel Prize Scientists Laboratory Project (C17213101), and SUSTech Special Fund for the Construction of High-Level Universities (No. G02216402).

\section{Author contributions}

P.-Y.J. and K.-F.F. performed experiments and prepared the supplementary information S.-H.X. helped with characterizing some new compounds. B.T. and S.L. conceived and directed the project and B.T., S.L., and S.-H.X. wrote the paper.

\section{Competing interests}

The authors declare no competing interests.

\section{Additional information}

Supplementary information The online version contains supplementary material available at https://doi.org/10.1038/s41467-021-22621-2.

Correspondence and requests for materials should be addressed to S.L. or B.T.

Peer review information Nature Communications thanks the anonymous reviewer(s) for their contribution to the peer review of this work.

Reprints and permission information is available at http://www.nature.com/reprints

Publisher's note Springer Nature remains neutral with regard to jurisdictional claims in published maps and institutional affiliations. 
(c) (i) Open Access This article is licensed under a Creative Commons Attribution 4.0 International License, which permits use, sharing, adaptation, distribution and reproduction in any medium or format, as long as you give appropriate credit to the original author(s) and the source, provide a link to the Creative Commons license, and indicate if changes were made. The images or other third party material in this article are included in the article's Creative Commons license, unless indicated otherwise in a credit line to the material. If material is not included in the article's Creative Commons license and your intended use is not permitted by statutory regulation or exceeds the permitted use, you will need to obtain permission directly from the copyright holder. To view a copy of this license, visit http://creativecommons.org/ licenses/by/4.0/.

(C) The Author(s) 2021 